\title{
ADI Schemes with Ikonen-Toivanen Splitting for Pricing American put Options in the Heston Model
}

\author{
Tinne Haentjens, Karel in 't Hout \& Kim Volders \\ Department of Mathematics and Computer Science, University of Antwerp, \\ Middelheimlaan 1, 2020 Antwerp, Belgium
}

\begin{abstract}
The numerical valuation of American put options under the Heston stochastic volatility model is considered. We investigate in this paper the potential of combining the recent splitting approach of Ikonen \& Toivanen $(2004$, 2009) with Alternating Direction Implicit schemes to obtain more efficient numerical methods.

Keywords: American options, Heston model, linear complementarity problems, finite difference methods, Ikonen-Toivanen splitting, ADI schemes.

PACS: $02.60 . \mathrm{Lj}, 02.70 . \mathrm{Bf}, 89.65 . \mathrm{Gh}$
\end{abstract}

\section{AMERICAN OPTION PRICE MODEL}

This paper deals with the numerical valuation of American put options under the popular Heston stochastic volatility model $[1,7]$ for the underlying asset price. We adapt the attractive numerical solution approach recently proposed by Ikonen \& Toivanen [5, 6] to the case where Alternating Direction Implicit (ADI) schemes are employed for the time discretization. We give evidence indicating that this can provide a further increase in efficiency.

Let $u(s, v, t)$ designate the fair price of an American put option under the Heston model if at time $T-t$ the asset price and its variance are equal to $s$ and $v$, respectively, where $T$ is the given maturity time of the option. The Heston operator applied to the function $u$ is given by

$$
\mathscr{A} u=\frac{1}{2} s^{2} v \frac{\partial^{2} u}{\partial s^{2}}+\rho \sigma s v \frac{\partial^{2} u}{\partial s \partial v}+\frac{1}{2} \sigma^{2} v \frac{\partial^{2} u}{\partial v^{2}}+r s \frac{\partial u}{\partial s}+\kappa(\eta-v) \frac{\partial u}{\partial v}-r u
$$

Here $\kappa>0$ is the mean-reversion rate, $\eta>0$ is the long-term mean, $\sigma>0$ is the volatility-of-variance, $\rho \in[-1,1]$ is the correlation between the two underlying Brownian motions, and $r$ is the interest rate. We shall assume $2 \kappa \eta>\sigma^{2}$ which is known as the Feller condition. If $K>0$ denotes the given exercise price, then the payoff function is

$$
\phi(s)=\max (K-s, 0) \quad(s \geq 0) .
$$

Financial option pricing theory yields that the function $u$ satisfies the so-called complementarity conditions

$$
u \geq \phi, \quad \frac{\partial u}{\partial t} \geq \mathscr{A} u, \quad(u-\phi)\left(\frac{\partial u}{\partial t}-\mathscr{A} u\right)=0,
$$

which are interpreted pointwise in the domain $s>0, v>0,0<t \leq T$; see e.g. [7]. Note that the second condition in (3) concerns a partial differential inequality. In numerical practice, a bounded spatial domain $[0, S] \times[0, V]$ is chosen with fixed values $S, V$ taken sufficiently large. We adjoin (3) with the same initial and boundary conditions from [6],

$$
u(s, v, 0)=\phi(s), \quad u(0, v, t)=K, \quad \frac{\partial u}{\partial s}(S, v, t)=0, \quad \frac{\partial u}{\partial v}(s, V, t)=0 .
$$

In view of the Feller condition, no boundary condition is needed at $v=0$.

\section{ADI SCHEMES WITH IKONEN-TOIVANEN SPLITTING}

For the numerical solution of the American put option pricing problem (3)-(4), first a spatial discretization of $\mathscr{A} u$ is performed. For ease of presentation we consider here a standard finite difference (FD) discretization on a Cartesian 
spatial grid $\left(s_{i}, v_{j}\right)$ with constant mesh widths $\Delta s=S / m_{1}$ and $\Delta v=V / m_{2}$ in the $s$ - and $v$-directions, respectively:

$$
\begin{aligned}
\left(u_{s}\right)_{i, j} & \approx \frac{u_{i+1, j}-u_{i-1, j}}{2 \Delta s} \\
\left(u_{v}\right)_{i, j} & \approx \frac{u_{i, j+1}-u_{i, j-1}}{2 \Delta v} \\
\left(u_{s s}\right)_{i, j} & \approx \frac{u_{i+1, j}-2 u_{i, j}+u_{i-1, j}}{(\Delta s)^{2}} \\
\left(u_{v v}\right)_{i, j} & \approx \frac{u_{i, j+1}-2 u_{i, j}+u_{i, j-1}}{(\Delta v)^{2}} \\
\left(u_{s v}\right)_{i, j} & \approx \frac{u_{i+1, j+1}+u_{i-1, j-1}-u_{i-1, j+1}-u_{i+1, j-1}}{4 \Delta s \Delta v}
\end{aligned}
$$

with the notation $u_{i, j}=u\left(s_{i}, v_{j}, t\right)$. Clearly, all of the above are second-order central FD formulas. The boundary conditions at $s=0, s=S$ and $v=V$ from (4) are easily incorporated into the FD discretization. At $v=0$, a secondorder upwind formula is used for the $u_{v}$ term. FD discretization yields the following, semi-discrete version of (3)-(4):

$$
U(t) \geq U_{0}, \quad U^{\prime}(t) \geq A U(t)+b, \quad\left(U(t)-U_{0}\right)^{T}\left(U^{\prime}(t)-A U(t)-b\right)=0 \quad(0<t \leq T),
$$

where inequalities are interpreted componentwise and ${ }^{T}$ denotes transpose. Here $A$ is a given real $m \times m$-matrix and $b, U_{0}$ are given real $m$-vectors with $m=m_{1}\left(m_{2}+1\right)$. The vector $U_{0}$ is determined by the payoff function $\phi$ and the vector $b$ depends on the boundary conditions. For each $t>0$, the entries of the solution vector $U(t)$ are approximations to the option prices $u(s, v, t)$ at the grid points $(s, v)=\left(s_{i}, v_{j}\right)$, ordered in a convenient way.

For the numerical solution of (5) we first discuss the prototype case of the Crank-Nicolson (CN) scheme. Let $I$ denote the $m \times m$ identity matrix and let $\Delta t=T / N$ with integer $N \geq 1$ be a given time step. Then approximations $U_{n} \approx U(n \cdot \Delta t)$ are defined for $n=1,2, \ldots, N$ by

$$
U_{n} \geq U_{0}, \quad\left(I-\frac{1}{2} \Delta t A\right) U_{n} \geq\left(I+\frac{1}{2} \Delta t A\right) U_{n-1}+\Delta t b, \quad\left(U_{n}-U_{0}\right)^{T}\left(\left(I-\frac{1}{2} \Delta t A\right) U_{n}-\left(I+\frac{1}{2} \Delta t A\right) U_{n-1}-\Delta t b\right)=0 .
$$

The discretization (6) is well-known in the literature, see e.g. Tavella \& Randall [7]. For each $n$ it constitutes a so-called linear complementarity problem (LCP). Ikonen \& Toivanen $[5,6]$ recently proposed the following splitting approach for the efficient solution of (6) :

$$
\begin{aligned}
& \left(I-\frac{1}{2} \Delta t A\right) \widetilde{U}_{n}=\left(I+\frac{1}{2} \Delta t A\right) U_{n-1}+\Delta t b+\Delta t \tilde{\lambda}_{n}, \\
& \left\{\begin{array}{c}
U_{n}-\widetilde{U}_{n}-\Delta t\left(\lambda_{n}-\tilde{\lambda}_{n}\right)=0, \\
U_{n} \geq U_{0}, \quad \lambda_{n} \geq 0, \quad\left(U_{n}-U_{0}\right)^{T} \lambda_{n}=0 .
\end{array}\right.
\end{aligned}
$$

The auxiliary vector $\tilde{\lambda}_{n}$ is given at the start of each step. We assume in this paper the basic choice

$$
\tilde{\lambda}_{n}=\lambda_{n-1}
$$

from loc. cit. with $\lambda_{0}$ the zero vector. Note that $U_{n}$ defined by (7)-(8) is actually an approximation to $U_{n}$ defined by (6). As we will not further consider method (6) by itself here, we suppress this in the notation. The derived method (7)-(8) has the useful feature that it is split in two convenient parts that can be handled in succession. The first part yields the internal vector $\widetilde{U}_{n}$ by solving a linear system that can be viewed as obtained from application of the CN scheme to a system of ordinary differential equations (ODEs). The second part yields the vectors $U_{n}, \lambda_{n}$ and are simply given by

$$
U_{n}=\max \left\{\widetilde{U}_{n}-\Delta t \lambda_{n-1}, U_{0}\right\}, \quad \lambda_{n}=\max \left\{0, \lambda_{n-1}+\left(U_{0}-\widetilde{U}_{n}\right) / \Delta t\right\},
$$

where the maximum is taken componentwise.

Similar applications of the above splitting approach were considered in $[5,6]$ for several other Runge-Kutta methods and linear multistep methods. The amount of computational work is determined by the solution of the linear systems in the different time steps and in the Heston case the authors employ a multigrid method for this. In the present paper 
we look into an alternative possibility, yielding simpler linear systems to solve in each time step, by choosing ADI type schemes for the time discretization. To this purpose, let the matrix $A$ be decomposed into

$$
A=A_{0}+A_{1}+A_{2},
$$

where the matrix $A_{0}$ corresponds to the mixed derivative term, $A_{1}$ corresponds to all spatial derivatives in the $s$ direction, and $A_{2}$ corresponds to all spatial derivatives in the $v$-direction, where the $r u$ term is distributed evenly over $A_{1}$ and $A_{2}$. Let $\theta>0$ be a given real parameter. For the numerical solution of (5), we consider adaptation of the Ikonen-Toivanen splitting approach to two known ADI type schemes.

The first is the Douglas (Do) scheme,

$$
\left\{\begin{array}{l}
Y_{0}=U_{n-1}+\Delta t\left(A U_{n-1}+b\right)+\Delta t \lambda_{n-1} \\
Y_{j}=Y_{j-1}+\theta \Delta t A_{j}\left(Y_{j}-U_{n-1}\right) \quad(j=1,2) \\
\widetilde{U}_{n}=Y_{2}
\end{array}\right.
$$

which is followed by (8) in each time step.

The second is the Modified Craig-Sneyd (MCS) scheme,

$$
\left\{\begin{array}{l}
Y_{0}=U_{n-1}+\Delta t\left(A U_{n-1}+b\right)+\Delta t \lambda_{n-1} \\
Y_{j}=Y_{j-1}+\theta \Delta t A_{j}\left(Y_{j}-U_{n-1}\right) \quad(j=1,2) \\
\widehat{Y}_{0}=Y_{0}+\theta \Delta t A_{0}\left(Y_{2}-U_{n-1}\right) \\
\widetilde{Y}_{0}=\widehat{Y}_{0}+\left(\frac{1}{2}-\theta\right) \Delta t A\left(Y_{2}-U_{n-1}\right) \\
\widetilde{Y}_{j}=\widetilde{Y}_{j-1}+\theta \Delta t A_{j}\left(\widetilde{Y}_{j}-U_{n-1}\right) \quad(j=1,2) \\
\widetilde{U}_{n}=\widetilde{Y}_{2}
\end{array}\right.
$$

which is followed by (8) in each time step.

In [2] various schemes of the ADI type were examined for the numerical valuation of European options in the Heston model, including the Do and MCS schemes. Comparing the Do and MCS schemes considered in the context of European options to their above versions, the only difference lies in the $\Delta t \lambda_{n-1}$ term in the first line. Much attention has been paid in [2] to the important case where the correlation $\rho$ is nonzero, so that an actual mixed derivative term is present, and it was found that the MCS scheme with parameter $\theta=\frac{1}{3}$ forms an effective method. The MCS scheme was introduced in [3] and possesses a classical order of consistency equal to two for ODEs for any $\theta$. The Do scheme is well-known in the literature, see e.g. [4] and the references therein. This scheme has a classical order of consistency equal to two for ODEs if and only if both $\theta=\frac{1}{2}$ and no mixed derivative term is present (i.e., $A_{0}$ is zero); otherwise the order reduces to one.

The schemes (9) and (10) retain the key advantage of traditional ADI schemes: the relevant linear systems are efficiently solved using $L U$ factorization, as the pertinent matrices are essentially tridiagonal. Note that this is in contrast to standard implicit methods, such as the $\mathrm{CN}$ scheme, where the pertinent matrices have a large bandwidth and solving by $L U$ factorization is in general too demanding.

Combination of ADI type schemes with the Ikonen-Toivanen splitting idea does not seem to have been considered in the literature so far. We gain first insight into its performance by a numerical experiment.

\section{NUMERICAL EXPERIMENT}

For the numerical experiment the same parameters for the Heston model and American put option are chosen as in [6]:

$$
\kappa=5, \quad \eta=0.16, \quad \sigma=0.9, \quad \rho=0.1, \quad r=0.1, \quad K=10, \quad T=0.25 .
$$




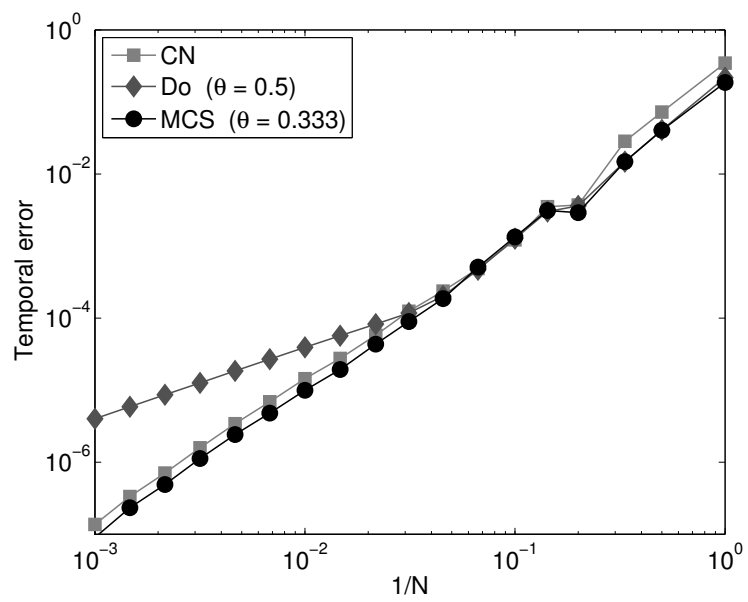

Further, $S=140$ and $V=5$ are taken to reduce the modeling error. We consider $m_{1}=100$ and $m_{2}=50$ mesh points in the $s$ - and $v$-directions, and study the global temporal error defined as the maximal absolute error between corresponding entries of the exact solution vector $U(T)$ to (5) and its approximation $U_{N}$ over the region of interest given by $\frac{1}{2} K<s_{i}<\frac{3}{2} K, 0<v_{j}<1$. We select three numerical methods for (5):

- the $\mathrm{CN}$ based method (7), (8)

- the Do based method (9), (8) with $\theta=\frac{1}{2}$

- the MCS based method (10), (8) with $\theta=\frac{1}{3}$.

For each of these methods, the figure displays the estimated global temporal errors vs. $1 / N$ for a range of step numbers between $N=1$ and $N=1000$. The $\mathrm{CN}$ scheme was applied with $N=3000$ to obtain a reference value for $U(T)$. The figure shows that, after a slightly erratic initial phase, the temporal errors for all three methods decay monotonically with increasing $N$. The observed order of convergence, for $N$ sufficiently large, is just 1.0 for the Do based method and 2.0 for both the $\mathrm{CN}$ and MCS based methods. Clearly, the obtained temporal errors for the latter two methods are essentially identical. Since the MCS based method requires much less computing effort per time step, we find that it is preferable over the $\mathrm{CN}$ based method in this experiment.

In view of the above, combination of ADI type schemes with the Ikonen-Toivanen splitting approach appears to be promising for arriving at more efficient methods for pricing American options in the Heston model. We shall investigate this in more detail in future research.

\section{ACKNOWLEDGEMENTS}

This work has been supported financially by the Research Foundation - Flanders, FWO contract no. G.0125.08 and no. 1.1.161.10.N.

\section{REFERENCES}

1. S. L. Heston, A closed-form solution for options with stochastic volatility with applications to bond and currency options, Rev. Finan. Stud. 6 (1993) 327-343.

2. K. J. in 't Hout \& S. Foulon, ADI finite difference schemes for option pricing in the Heston model with correlation, Int. J. Numer. Anal. Mod. 7 (2010) 303-320.

3. K. J. in 't Hout \& B. D. Welfert, Unconditional stability of second-order ADI schemes applied to multi-dimensional diffusion equations with mixed derivative terms, Appl. Numer. Math. 59 (2009) 677-692.

4. W. Hundsdorfer \& J. G. Verwer, Numerical Solution of Time-Dependent Advection-Diffusion-Reaction Equations, Springer, Berlin, 2003.

5. S. Ikonen \& J. Toivanen, Operator splitting methods for American option pricing, Appl. Math. Lett. 17 (2004) 809-814.

6. S. Ikonen \& J. Toivanen, Operator splitting methods for pricing American options under stochastic volatility, Numer. Math. 113 (2009) 299-324.

7. D. Tavella \& C. Randall, Pricing Financial Instruments, Wiley, New York, 2000. 